\title{
Erratum: Who's who in biotech
}

Kendall Powell

Nat. Biotechnol. 24, 294 (2006)

In the print version of this article and the version originally published online, there is an error. On page 294, the first name of Dr. Potrykus should read "Ingo" and not "Ingro."

\section{Erratum: Who's who in biotech}

\section{Cormac Sheridan}

Nat. Biotechnol. 24, 291-300 (2006)

In the print version of this article and the version originally published online, there is an error. On page 296, the name of the CEO of Novartis was misspelled as "Vassella"; the correct spelling is "Vasella."

\section{Erratum: Who's who in biotech}

\section{K.S. Jayaraman}

Nat. Biotechnol. 24, 291-300 (2006)

In the print version of this article and the version originally published online, there is an error. On page 299, left column, second paragraph, it should have said that C.S. Prakash has been involved in AgBioWorld (http://www.agbioworld.org/) and not AgBioForum as originally stated.

\section{Erratum: Gene prioritization through genomic data fusion}

Stein Aerts, Diether Lambrechts, Sunit Maity, Peter Van Loo, Bert Coessens, Frederik De Smet, Leon-Charles Tranchevent, Bart De Moor, Peter Marynen, Bassem Hassan, Peter Carmeliet \& Yves Moreau

Nat. Biotechnol. 24, 537-544 (2006)

In the print version of this article and the version originally published online, reference 5 was mistakenly included on page 537 , column 2 , lines 3 , 10 and reference 1 on line 17, and the line "Ouzounis ${ }^{8}$ and the Prospectr scores from Euan Adie" was repeated in the acknowledgments on page 544.

\section{Corrigendum: Conflict resolution in the biotech industry}

Mary Yaroshevsky-Glanville

Nat. Biotechnol. 23, 391-392 (2005)

It has been brought to the attention of the author and the editors that in the print version of this article and the version originally published online, Andrea Corney, of Acorn Consulting, should have been credited with the situations described on page 391, third paragraph, and the eight commonalities listed as bullet points on page 391, second column. They initially appeared in an online newsletter, http://www.acorn-od.com/ shorts/archive/shorts-03.html. 